\title{
On the holomorphically projective correspondence between Kählerian spaces preserving complex structure
}

\author{
By Toshio SAKaguchi
}

\section{Introduction.}

Let $\left(M^{n}, g\right)$ and $\left(M^{\prime n}, g^{\prime}\right)(n>2)$ be $n$-dimensional Riemannian spaces with positive definite metric tensors $g$ and $g^{\prime}$, respectively. The celebrated Beltrami's theorem (see $[1]^{1)} \S 40$ ) of classical differential geometry states that if $\left(M^{n}, g\right)$ is of constant curvature then the space which is in projective correspondence with it is necessarily of constant curvature. N. S. Sinyukov [5] has generalized the above theorem to the non-affine projective correspondence of $\left(M^{n}, g\right)$ with $\left(M^{\prime n}, g^{\prime}\right)$ which is locally symmetric. Further W. Roter [4] and J. Robinson and J. D. Zund [3] have generalized N. S. Sinyukov's theorem to the case when $\left(M^{\prime n}, g^{\prime}\right)$ is recurrent.

On the other hand T. Ōtsuki and Y. Tashiro [2] have studied holomorphically projective correspondences between Kählerian spaces. Let $\left(M^{n}\right.$, $g, F)$ and $\left(M^{\prime n}, g^{\prime}, F^{\prime}\right)$ be Kählerian spaces of real dimension $n(=2 m>2)$ with the structures $(g, F)$ and $\left(g^{\prime}, F^{\prime}\right)$, respectively. Then they have proved the generalization of Beltrami's theorem, that is, if $\left(M^{n}, g, F\right)$ is of constant holomorphic sectional curvature then the space which is in holomorphically projective correspondence with it preserving complex structure is necessarily of constant holomorphic sectional curvature.

The purpose of the present paper is to investigate what restrictions the requirement that $\left(M^{n}, g, F\right)$ and $\left(M^{\prime n}, g^{\prime}, F^{\prime}\right)$ be in holomorphically projective correspondence preserving complex structure imposes on these spaces. An example of such a restriction is given by the above result of T. Ötsuki and Y. Tashiro.

The author wishes to express here his sincere thanks to Professor Yoshie Katsurada and Doctor Tamao Nagai for their kindly guidances and advices.

\section{§1. Preliminaries.}

We shall first give preliminary formulas on the Kählerian space and the holomorphically projective correspondence between Kählerian spaces.

1) Numbers in brackets refer to the references at the end of the paper. 
Let us consider an $n(=2 m>2)$ real dimensional Kählerian space with local coordinates $\left\{x^{i}\right\}$. Then the positive definite Riemannian metric $g_{j i}$ and the complex structure $F_{i}^{h}$ satisfy the following equations

$$
\begin{array}{cc}
F_{j}{ }^{a} F_{a}{ }^{i}=-\delta_{j}{ }^{i}, & g_{a b} F_{j}{ }^{a} F_{i}^{b}=g_{j i}, \\
\nabla_{k} F_{j}{ }^{h}=0, & \nabla_{k} g_{j i}=0,
\end{array}
$$

where $\nabla_{k}$ denotes the operator of the covariant differentiation with respect to the Christoffel symbols $\left\{\begin{array}{l}h \\ j i\end{array}\right\}$ constructed from $g_{j i}$.

The Riemannian curvature tensor of $g_{j i}$ is defined by

$$
R_{k j i}{ }^{h}=\partial_{k}\left\{\begin{array}{c}
h \\
j i
\end{array}\right\}-\partial_{j}\left\{\begin{array}{c}
h \\
k i
\end{array}\right\}+\left\{\begin{array}{c}
h \\
k a
\end{array}\right\}\left\{\begin{array}{c}
a \\
j i
\end{array}\right\}-\left\{\begin{array}{c}
h \\
j a
\end{array}\right\}\left\{\begin{array}{c}
a \\
k i
\end{array}\right\}, \quad \partial_{j}=\partial / \partial x^{j} .
$$

We put $R_{k j t h}=R_{k j i}^{a} g_{a h}, R_{j i}=R_{a j i}{ }^{a}=R_{a j i b} g^{a b}, R=R_{a b} g^{a b}$ and

$$
S_{j i}=F_{j}^{a} R_{a i},
$$

then the following identities are valid

$$
\begin{aligned}
& R_{a j i}{ }^{h} F_{k}^{a}=-R_{k a i}{ }^{h} F_{j}^{a}, \quad R_{k j a}{ }^{h} F_{i}^{a}=R_{k j i}{ }^{a} F_{a}{ }^{n}, \\
& R_{k j i h}=R_{k j a b} F_{i}^{a} F_{h}^{b} \text {, } \\
& R_{j i}=R_{a b} F_{j}{ }^{a} F_{i}^{b}, \quad F_{j}{ }^{a} R_{a i}=-R_{j a} F_{i}{ }^{a}, \quad F_{j}{ }^{a} R_{a}{ }^{h}=R_{j}{ }^{a} F_{a}{ }^{h}, \\
& S_{j i}+S_{i j}=0, \quad S_{j i}=S_{a b} F_{j}{ }^{a} F_{i}^{b}, \quad F_{j}{ }^{a} S_{a i}=-S_{j a} F_{i}{ }^{a}=-R_{j i}, \\
& S_{j i}=-\frac{1}{2} F^{a b} R_{j i a b}=F^{a b} R_{a j i b} .
\end{aligned}
$$

The holomorphically projective curvature tensor $P_{k j i}{ }^{h}$ is given by

$$
P_{k j i}{ }^{h}=R_{k j i}{ }^{h}+\frac{1}{n+2}\left(R_{k i} \delta_{j}{ }^{n}-R_{j i} \delta_{k}{ }^{h}+S_{k i} F_{j}{ }^{h}-S_{j i} F_{k}{ }^{h}+2 S_{k j} F_{i}{ }^{h}\right) .
$$

We can obtain easily the following identities

$$
\begin{gathered}
P_{k j i}{ }^{h}+P_{j k i}{ }^{h}=0, \quad P_{k j i}{ }^{h}+P_{j i k}{ }^{n}+P_{i k j}{ }^{h}=0, \quad P_{a j i}{ }^{a}=0, \\
P_{k j i}{ }^{a} F_{a}{ }^{h}=P_{k j a}{ }^{n} F_{i}{ }^{a}, \quad P_{a j i}{ }^{n} F_{k}{ }^{a}=P_{a k i}{ }^{h} F_{j}{ }^{a},
\end{gathered}
$$

from which we get

$$
P_{k j a}{ }^{a}=0, \quad P_{a j i}^{b} F_{b}^{a}=0, \quad P_{k j a b}^{b} F_{b}^{a}=0 .
$$

A necessary and sufficient condition for $P_{k j i}{ }^{n}=0$ is that the space is of constant holomorphic sectional curvature, i.e., a space whose curvature tensor $R_{k j i}^{h}$ takes the form

$$
R_{k j i}{ }^{h}=k\left(g_{k i} \delta_{j}{ }^{h}-g_{j i} \delta_{k}{ }^{h}+F_{k i} F_{j}{ }^{h}-F_{j i} F_{k}{ }^{h}+2 F_{k j} F_{i}{ }^{h}\right),
$$

where we put 


$$
k=-\frac{R}{n(n+2)} .
$$

Let $\left(M^{n}, g, F\right)$ and $\left(M^{\prime n}, g^{\prime}, F^{\prime}\right)$ be Kählerian spaces of real dimension $n(=2 m>2)$ with the structures $(g, F)$ and $\left(g^{\prime}, F^{\prime}\right)$, respectively. A necessary and sufficient condition for a correspondence $f$ from $\left(M^{n}, g, F\right)$ to $\left(M^{\prime n}, g^{\prime}\right.$, $F^{\prime}$ ) to be holomorphically projective is that there exists a vector field $\phi_{i}$ defined on $\left(M^{n}, g, F\right)$ such that

$$
\left\{\begin{array}{c}
\bar{h} \\
j i
\end{array}\right\}=\left\{\begin{array}{c}
h \\
j i
\end{array}\right\}+\phi_{j} \delta_{i}{ }^{h}+\phi_{i} \delta_{j}{ }^{h}-\bar{\psi}_{j} F_{i}{ }^{h}-\bar{\psi}_{i} F_{j}{ }^{n},
$$

where $\left\{\begin{array}{c}\bar{h} \\ j i\end{array}\right\}$ is the Christoffel symbols constructed from $\bar{g}=f^{*} g^{\prime}$ and $\bar{\psi}_{i}=$ $\phi_{a} F_{i}{ }^{a}$.

From (1.7) we know that $\phi_{i}$ is necessarily gradient. Especially if $\phi_{i}=0$, then the correspondence is said to be affine.

For convenience' sake we put "- " over the quantities induced from the quantities on $\left(M^{\prime n}, g^{\prime}, F^{\prime}\right)$ by $f$. From (1.7) we have

(1.8) $\quad \bar{R}_{k j i}{ }^{h}=R_{k j i}{ }^{h}+\phi_{k i} \delta_{j}^{h}-\psi_{j i} \delta_{k}{ }^{h}-\left(\psi_{k a} F_{j}^{h}-\psi_{j a} F_{k}{ }^{h}\right) F_{i}^{a}-\left(\psi_{k a} F_{j}^{a}-\psi_{j a} F_{k}^{a}\right) F_{i}^{h}$, where $\psi_{j i}$ is a symmetric tensor defined by

$$
\phi_{j i}=\nabla_{j} \psi_{i}-\phi_{j} \phi_{i}+\bar{\psi}_{j} \bar{\psi}_{i} .
$$

Contracting $h$ and $k$ in (1.8), we get

$$
\bar{R}_{j i}=R_{j i}-n \psi_{j i}-2 F_{j}^{a} F_{i}^{b} \psi_{a b} .
$$

We assume henceforth that the holomorphically projective correspondence preserves the complex structure, i. e., $f^{*} F^{\prime}=\bar{F}=F$. Then we have

$$
\overline{\boldsymbol{F}}_{i}{ }^{j}=F_{i}{ }^{j}, \quad \overline{\boldsymbol{D}}_{k} F_{i}^{j}=0,
$$

where $\bar{\nabla}_{k}$ denotes the operator of the covariant differentiation with respect to $\left\{\begin{array}{c}\bar{h} \\ j i\end{array}\right\}$.

On the other hand we know that $R_{j i}$ and $\vec{R}_{j i}$ are hybrid by (1.10), i. e.,

$$
R_{j i}=R_{a b} F_{j}^{a} F_{i}^{b}, \quad \bar{R}_{j i}=\bar{R}_{a b} F_{j}^{a} F_{i}^{b} .
$$

Taking use of this fact, we obtain by means of (1.9)

$$
(n-2)\left(\phi_{j i}-\phi_{a b} F_{j}^{a} F_{i}^{b}\right)=0,
$$

which implies because of $n>2$ that $\phi_{j t}$ is hybrid, i.e.,

$$
\phi_{j i}=\phi_{a b} F_{j}^{a} F_{i}^{b} .
$$

Substituting (1.11) into (1.8) and (1.9) we find 


$$
\begin{gathered}
\bar{R}_{k j i}{ }^{h}=R_{k j i}{ }^{h}+\psi_{k i} \delta_{j}{ }^{h}-\psi_{j i} \delta_{k}{ }^{n}-\bar{\psi}_{k i} F_{j}{ }^{h}+\bar{\psi}_{j i} F_{k}{ }^{h}-2 \bar{\psi}_{k j} F_{i}{ }^{h}, \\
\bar{R}_{j i}=R_{j i}-(n+2) \phi_{j i},
\end{gathered}
$$

where $\tilde{\psi}_{j i}$ is a skew-symmetric tensor defined by

$$
\tilde{\psi}_{j i}=\phi_{j a} F_{i}^{a}=\nabla_{j} \tilde{\psi}_{i}-\phi_{j} \bar{\psi}_{i}-\bar{\psi}_{j} \psi_{i} .
$$

From (1.11) we have that $\tilde{\psi}_{j i}$ is hybrid, i.e.,

$$
\tilde{\psi}_{j i}=\tilde{\psi}_{a b} F_{j}{ }^{a} F_{i}^{b} \text {. }
$$

By transvecting (1.13) with $F_{k}^{j}$ we obtain

$$
\overline{\mathbf{S}}_{k i}=S_{k i}+(n+2) \tilde{\psi}_{k i},
$$

where $\bar{S}_{k i}$ is a skew-symmetric tensor defined by

$$
\bar{S}_{k i}=\bar{F}_{k}^{a} \bar{R}_{a i}=F_{k}^{a} \bar{R}_{a i} \text {. }
$$

Kählerian space is said to be recurrent if there exists a vector field $K_{l}$ satisfying the following identity

$$
\nabla_{l} R_{k j i}^{h}=K_{l} R_{k j i}^{h} .
$$

In this case, we shall call $K_{l}$ the recurrent vector. We know that the recurrent vector $K_{l}$ is locally a gradient one. If $K_{l}=0$, then a recurrent Kählerian space is said to be locally symmetric.

\section{§2. A holomorphically projective correspondence preserving complex structure.}

In this section we shall prove the following

THEOREM. Let $\left(M^{n}, g, F\right)(n>2)$ be an arbitrary Kählerian space and $\left(M^{\prime n}, g^{\prime}, F^{\prime}\right)$ a recurrent Kählerian space. If there exists a non-affine holomorphically projective correspondence between $\left(M^{n}, g, F\right)$ and $\left(M^{\prime n}, g^{\prime}, F^{\prime}\right)$ preserving complex structure, $\left(M^{n}, g, F\right)$ and $\left(M^{\prime n}, g^{\prime}, F^{\prime}\right)$ are of constant holomorphic sectional curvature.

We shall first prove the following

Lemma. Let $\left(M^{n}, g, F\right)$ and $\left(M^{\prime n}, g^{\prime}, F^{\prime}\right)(n>2)$ be two Kählerian spaces such that there exists a holomorphically projective correspondence between $\left(M^{n}, g, F\right)$ and $\left(M^{\prime n}, g^{\prime}, F^{\prime}\right)$ preserving complex structure. If $\left(M^{\prime n}, g^{\prime}, F^{\prime}\right)$ satisfies

$$
\nabla_{m}^{\prime} \nabla_{l}^{\prime} R_{i j k}^{\prime}{ }^{h}-\nabla_{i}^{\prime} \nabla_{m}^{\prime} R_{i j k}^{\prime h}=0,
$$

where $\nabla_{k}^{\prime}$ denotes the operator of the covariant differentiation with respect to $\left\{\begin{array}{l}h \\ j i\end{array}\right\}^{\prime}$ constructed from $g^{\prime}$ and $R_{i j k}^{\prime}$ the Riemannian curvature tensor of 
$g_{j i}^{\prime}$, then one of the following two conditions must be satisfied.

$$
\begin{aligned}
& R_{i j}-\frac{1}{n} R g_{i j}=0 . \\
& \Delta g_{i j}-n \psi_{i j}=0,
\end{aligned}
$$

where $\Delta=g^{a b} \psi_{a b}$.

PROOF. From our assumption we have

$$
\bar{\nabla}_{m} \bar{\nabla}_{l} \bar{R}_{i j k}^{h}-\bar{\nabla}_{l} \bar{\nabla}_{m} \bar{R}_{i j k}{ }^{h}=0 .
$$

By the Ricci's identity we find

$$
\bar{R}_{a j k}{ }^{h} \bar{R}_{m l i}^{a}+\bar{R}_{i a k}{ }^{h} \bar{R}_{m l j}{ }^{a}+\bar{R}_{i j a}{ }^{h} \bar{R}_{m l k}{ }^{a}-\bar{R}_{i j k}{ }^{a} \bar{R}_{m l a}{ }^{h}=0 .
$$

Using (1.12) we now replace $\bar{R}_{k j i}{ }^{h}$ by $R_{k j i}{ }^{h}$, transvect the result with $g^{i t}$ and lower the index $h$. The symmetric part in the indices $m, j$ and $k, h$ of its result can be written as

$$
Q_{h j k m}+Q_{k j h m}=0
$$

where

$$
\begin{aligned}
Q_{h j k m}= & g_{h j} \psi_{k a} R_{m}{ }^{a}+g_{k m} \psi_{h a} R_{j}{ }^{a}-R_{h j} \psi_{k m} \\
& -R_{k m} \psi_{h j}-F_{h j} \psi_{k a} S_{m}{ }^{a}-F_{k m} \psi_{h a} S_{j}{ }^{a}+S_{h j} \tilde{\psi}_{k m}+S_{k m} \tilde{\psi}_{h j},
\end{aligned}
$$

after some complicated calculation.

Contracting $i$ and $h$ in (2.1), we have

$$
\bar{\nabla}_{m} \bar{\nabla}_{l} \bar{R}_{j k}-\bar{\nabla}_{l} \bar{\nabla}_{m} \bar{R}_{j k}=0 \text {. }
$$

By the Ricci's identity we find

$$
\bar{R}_{a k} \bar{R}_{m l j}{ }^{a}+\bar{R}_{j a} \bar{R}_{m l k}{ }^{a}=0 .
$$

Using (1.12) and (1.13) we replace $\bar{R}_{k j i}{ }^{h}$ and $\bar{R}_{k j}$ by $R_{k j i}{ }^{h}$ and $R_{k j}$ and transvect the result with $g^{j k}$. Then we have

$$
\phi_{m a} R_{l}{ }^{a}=\phi_{l a} R_{m}^{a},
$$

after some calculation.

Transvecting (2.4) with $F_{k}^{l}$, we obtain

$$
\psi_{m a} S_{k}{ }^{a}=-\psi_{k a} S_{m}{ }^{a} .
$$

We put $Q_{h j k m}=A_{h j k m}+B_{h j k m}$,

where

$$
\begin{aligned}
& A_{k j k m}=g_{h j} \psi_{k a} R_{m}{ }^{a}+g_{k m} \psi_{h a} R_{j}{ }^{a}-R_{h j} \psi_{k m}-R_{k m} \psi_{h j}, \\
& B_{h j k m}=-F_{h j} \psi_{k a} S_{m}{ }^{a}-F_{k m} \psi_{h a} S_{j}{ }^{a}+S_{h j} \bar{\psi}_{k m}+S_{k m} \bar{\psi}_{h j} .
\end{aligned}
$$


By (2.4) and (2.5) we have

$$
\begin{aligned}
& A_{h j k m}=A_{j h k m}=A_{k m h j}, \\
& B_{h j k m}=-B_{j h k m}=B_{k m h j} .
\end{aligned}
$$

By a straight computation, we obtain

$$
F_{h}{ }^{a} F_{j}^{b} Q_{a b k m}=Q_{h j k m}, \quad F_{j}^{a} F_{k}^{b} Q_{h a b m}=-Q_{h j k m} .
$$

From (2.2) and these equations we get

$$
0=F_{h}{ }^{a} F_{j}{ }^{b}\left(Q_{a b k m}+Q_{k b a m}\right)=Q_{h j k m}-Q_{k j h m} .
$$

Hence, from (2.2) we obtain

$$
Q_{h j k m}=0
$$

From (2.6) we have

$$
0=Q_{h j k m} A^{h j k m}=A_{h j k m} A^{h j k m} .
$$

Hence we get

$$
A_{h j k m}=g_{h j} \psi_{k a} R_{m}{ }^{a}+g_{k m} \psi_{h a} R_{j}{ }^{a}-R_{h j} \psi_{k m}-R_{k m} \psi_{h j}=0 .
$$

Transvecting (2.7) with $g^{h j}$, we obtain

$$
n \psi_{k a} R_{m}{ }^{a}+g_{k m} \psi_{a b} R^{a b}-R \psi_{k m}-\Delta R_{k m}=0,
$$

which upon further transvection with $g^{k m}$ yields

$$
n \psi_{a b} R^{a b}=\Delta R \text {. }
$$

The last two equations enable us to write (2.7) in the following convenient form

$$
\left(R_{h j}-\frac{R}{n} g_{h j}\right)\left(\Delta g_{k m}-n \psi_{k m}\right)+\left(R_{k m}-\frac{R}{n} g_{k m}\right)\left(\Delta g_{h j}-n \psi_{h j}\right)=0,
$$

which clearly means that either

$$
R_{i j}-\frac{R}{n} g_{i j}=0,
$$

or

$$
\Delta g_{i j}-n \psi_{i j}=0 . \quad \text { Q.E. D. }
$$

Proof of THE THEOREM.

Since $\left(M^{\prime n}, g^{\prime}, F^{\prime}\right)$ is recurrent, we have by definition

$$
\nabla_{l}^{\prime} R_{i j k}^{\prime}{ }^{h}=K_{l}^{\prime} R_{i j k}^{\prime}{ }^{h} .
$$

Let us consider the integrability conditions of (2.8):

$$
\nabla_{m}^{\prime} \nabla_{l}^{\prime} R_{i j k}^{\prime}{ }^{n}-\nabla_{l}^{\prime} \nabla_{m}^{\prime} R_{i j k}^{\prime}{ }^{n}=\left(\nabla_{m}^{\prime} K_{l}^{\prime}-\nabla_{l}^{\prime} K_{m}^{\prime}\right) R_{i j k}^{\prime}{ }^{n}+K_{l}^{\prime} \nabla_{m}^{\prime} R_{i j k}^{\prime}-K_{m}^{\prime} \nabla_{l}^{\prime} R_{i j k}^{\prime}{ }^{n} .
$$


Since $K_{\imath}^{\prime}$ is locally a gradient vector and by (2.8), the right-hand of the last equation vanishes. Hence, by the lemma we have one of the following two cases.

$$
\begin{array}{ll}
\text { Case A : } & R_{i j}-\frac{R}{n} g_{i j}=0 . \\
\text { Case B : } & \Delta g_{i j}-n \psi_{i j}=0 .
\end{array}
$$

Let us consider first the case A. By $(2.8)$ we have

$$
\bar{\nabla}_{l} \bar{R}_{i j k}^{h}=\bar{K}_{l} \bar{R}_{i j k}^{h}, \quad \bar{\nabla}_{l} \bar{R}_{j k}=\bar{K}_{l} \bar{R}_{j k}, \quad \bar{\nabla}_{l} \bar{S}_{i j}=\bar{K}_{l} \bar{S}_{i j}
$$

and hence

$$
\bar{\nabla}_{l} \bar{P}_{i j k}^{h}=\bar{K}_{\imath} \bar{P}_{i j k}^{h} .
$$

By construction the holomorphically projective curvature tensor is invariant under a holomorphically projective correspondence and hence in the last equation we can replace $\bar{P}_{i j k}^{h}$ by $P_{i j k}{ }^{n}$, i. e.,

$$
\bar{\nabla}_{l} P_{i j k}{ }^{h}=\bar{K}_{l} P_{i j k}{ }^{h} .
$$

This equation may be written out explicity giving

$$
\begin{aligned}
& \nabla_{l} P_{i j k}{ }^{n}+\psi_{a} P_{i j k}{ }^{a} \delta_{l}{ }^{n}-\bar{\psi}_{a} P_{i j k}{ }^{a} F_{l}{ }^{h}-2 \psi_{l} P_{i j k}{ }^{n}-\psi_{i} P_{l j k}{ }^{n}-\psi_{j} P_{i l k}{ }^{n} \\
& -\psi_{k} P_{i j l}{ }^{n}+\left(\tilde{\psi}_{i} P_{a j k}{ }^{h}+\tilde{\psi}_{j} P_{i a k}{ }^{h}+\bar{\psi}_{k} P_{i j a}{ }^{h}\right) F_{l}^{a}=\bar{K}_{l} P_{i j k}{ }^{n} .
\end{aligned}
$$

If we lower the index $h$ and symmetrize the result over $k$ and $h$, we find that

$$
\begin{gathered}
\psi_{a} P_{i j k}{ }^{a} g_{l h}+\psi_{a} P_{i j h}{ }^{a} g_{l k}-\bar{\psi}_{a} P_{i j k}{ }^{a} F_{l h}-\tilde{\psi}_{a} P_{i j h}{ }^{a} F_{l k}-\psi_{k} P_{i j l h} \\
-\psi_{h} P_{i j l k}+\bar{\psi}_{k} P_{i j a h} F_{l}^{a}+\tilde{\psi}_{h} P_{i j a k} F_{l}^{a}=0,
\end{gathered}
$$

since in the case A $P_{i j k n}$ is skew-symmetric with respect to $k$ and $h$. By transvecting (2.9) with $g^{l h}$ and taking use of (1.5) and (1.6), we find that $\phi_{a} P_{i j k}{ }^{a}=0$ and $\tilde{\varphi}_{a} P_{i j k}{ }^{a}=0$ which allow us to reduce (2.9) to

$$
-\psi_{k} P_{i j l h}-\psi_{h} P_{i j l k}+\bar{\psi}_{k} P_{i j a h} F_{l}^{a}+\tilde{\psi}_{h} P_{i j a k} F_{l}^{a}=0 .
$$

By transvecting (2.10) with $\psi^{k} P^{i j l h}$ we find that

$$
\left(\phi_{k} P_{i j l \hbar}\right)\left(\phi^{k} P^{i j l h}\right)=0 \text {. }
$$

Since we are considering only non-affine holomorphically projective correspondence, we have clearly $P_{i j l h}=0$, which implies that $\left(M^{n}, g, F\right)$ is of constant holomorphic sectional curvature. But since the holomorphically projective curvature tensor is invariant under holomorphically projective correspondence, $\bar{P}_{i j k}{ }^{n}=0$ and hence in the case A, $\left(M^{\prime n}, g^{\prime}, F^{\prime}\right)$ is also of constant holomorphic sectional curvature.

Consider now the case $\mathrm{B}$, and let 


$$
\Pi_{i j k}{ }^{h}=R_{i j k}{ }^{h}+\frac{\Delta}{n}\left(g_{i k} \delta_{j}{ }^{h}-g_{j k} \delta_{i}{ }^{h}+F_{i k} F_{j}{ }^{h}-F_{j k} F_{i}{ }^{h}+2 F_{i j} F_{k}{ }^{h}\right) .
$$

Using (2.11) we see that

$$
\Pi_{i j k}{ }^{h}=\bar{R}_{i j k}{ }^{h}, \quad \bar{\nabla}_{i} \Pi_{i j k}{ }^{h}=\bar{K}_{l} \Pi_{i j k}{ }^{h} .
$$

By a straight computation, we have

$$
\begin{gathered}
\Pi_{i j k}{ }^{a} g_{a h}=\Pi_{i j k h}=-\Pi_{j i k h}=-\Pi_{i j h k}, \quad \Pi_{i j k h}=\Pi_{k h i j}, \\
\Pi_{i j k h}+\Pi_{j k i h}+\Pi_{k i j h}=0, \\
\Pi_{a j k}{ }^{a}=R_{j k}-(n+2) \phi_{j k}, \quad \Pi_{i j a}{ }^{a}=0, \\
\Pi_{i j k}{ }^{a} F_{a}{ }^{h}=\Pi_{i j a}{ }^{b} F_{k}^{a}, \quad \Pi_{a j k}{ }^{a} F_{i}^{a}=-\Pi_{i a k}{ }^{b} F_{j}{ }^{a}, \\
\Pi_{i j a}{ }^{b} F_{b}^{a}=2\left\{S_{i j}+(n+2) \tilde{\psi}_{i j}\right\}, \quad \Pi_{a j k}{ }^{b} F_{b}^{a}=-\left\{S_{j k}+(n+2) \tilde{\psi}_{j k}\right\}, \\
F^{i j} \Pi_{i j a}^{b} F_{b}^{a}=R-(n+2) \Delta .
\end{gathered}
$$

From the second equation of (2.12) and (2.13) we get

$$
\begin{aligned}
& \nabla_{l} \Pi_{i j k}{ }^{h}+\psi_{a} \Pi_{i j k}{ }^{a} \delta_{l}^{h}-\bar{\psi}_{a} \Pi_{i j k}{ }^{a} F_{l}^{h}-2 \psi_{l} \Pi_{i j k}{ }^{h}-\psi_{i} \Pi_{l j k}{ }^{h}-\psi_{j} \Pi_{i l k}{ }^{h} \\
& -\psi_{k} \Pi_{i j l}{ }^{h}+\left(\bar{\psi}_{i} \Pi_{a j k}{ }^{h}+\bar{\psi}_{j} \Pi_{i a k}{ }^{h}+\bar{\psi}_{k} \Pi_{i j a}{ }^{h}\right) F_{l}{ }^{a}=\bar{K}_{l} \Pi_{i j k}{ }^{h} .
\end{aligned}
$$

If we lower the index $h$ and symmetrize the result over $k$ and $h$, taking use of the first equation of (2.13) we get

$$
\begin{aligned}
\phi_{a} \Pi_{i j k}{ }^{a} g_{l h}+ & \psi_{a} \Pi_{i j h}{ }^{a} g_{l k}-\tilde{\psi}_{a} \Pi_{i j k}{ }^{a} F_{l h}-\tilde{\psi}_{a} \Pi_{i j h}{ }^{a} F_{l k} \\
& -\psi_{k} \Pi_{i j l h}-\psi_{h} \Pi_{i j l k}+\tilde{\psi}_{k} \Pi_{i j a h} F_{l}{ }^{a}+\tilde{\psi}_{h} \Pi_{i j a k} F_{l}{ }^{a}=0 .
\end{aligned}
$$

By transvecting (2.15) with $g^{\text {lh }}$ and taking use of (2.13), we obtain

$$
n \psi_{a} \Pi_{i j k}{ }^{a}+\tilde{\psi}_{k} \Pi_{i j a}{ }^{b} F_{b}{ }^{a}=0 .
$$

Transvecting (2.16) with $\phi_{c} \Pi^{i j k c}$ we have

$$
n\left(\psi_{a} \Pi_{i j k}^{a}\right)\left(\psi_{c} \Pi^{i j k c}\right)+\psi_{c} \Pi_{i j k}{ }^{c} \bar{\psi}^{k} \Pi^{i j}{ }_{a}^{b} F_{b}{ }^{a}=0 .
$$

If we can show that $\psi_{c} \Pi_{i j k}{ }^{c} \bar{\psi}^{k} \Pi^{i j}{ }_{a}^{b} F_{b}{ }^{a}=0$, then we have $\phi_{a} \Pi_{i j k}{ }^{a}=0$ and $\bar{\psi}_{a} \Pi_{i j k}{ }^{a}=0$ which allow us to reduce (2.15) to

$$
-\psi_{k} \Pi_{i j l h}-\psi_{h} \Pi_{i j l k}+\tilde{\psi}_{k} \Pi_{i j a h} F_{l}^{a}+\tilde{\psi}_{h} \Pi_{i j a k} F_{l}^{a}=0 \text {. }
$$

Then we can repeat the argument which was previously used in the case A to deduce that $\Pi_{i j k h}=0$. Hence, from the first equation of (2.12) this means that $\left(M^{\prime n}, g^{\prime}, F^{\prime}\right)$ is flat Kählerian space and by $(2.11)$ we see that $\left(M^{n}, g, F\right)$ is of constant holomorphic sectional curvature... By (2.11) in this case the holomorphically projective correspondence preserving complex structure must satisfy $\Delta=$ constant.

Now we shall prove that $\psi_{c} \Pi_{i j k}{ }^{c} \bar{\psi}^{k} \Pi^{i j}{ }_{a}^{b} F_{b}{ }^{a}=0$. Transvecting (2.16) 
with $F^{i j}$ and taking use of (2.13), we obtain

$$
n \psi_{a}\left\{S_{k}{ }^{a}+(n+2) \tilde{\psi}_{k}^{a}\right\}-\tilde{\psi}_{k}\{R-(n+2) \Delta\}=0 \text {. }
$$

Transvecting (2.17) with $\tilde{\psi}^{k}$ and taking use of $\tilde{\psi}^{a} \tilde{\psi}_{a}=\phi^{a} \psi_{a}$ w whe

$$
n \bar{\psi}^{k} \psi_{a}\left\{S_{k}^{a}+(n+2) \tilde{\psi}_{k}^{a}\right\}-\phi^{a} \psi_{a}\{R-(n+2) \Delta\}=0 \text {. }
$$

Substituting (2.16) into $-\frac{n}{2} \psi_{a}\left(\Pi_{i j k}{ }^{a}+\Pi_{j k i}{ }^{a}+\Pi_{k i j}{ }^{a}\right)=0$ and transvecting the result with $F^{i j} \bar{\psi}^{k}$, we obtain

$$
\phi^{a} \psi_{a}\{R-(n+2) \Delta\}-2 \tilde{\psi}^{k} \psi_{a}\left\{S_{k}^{a}+(n+2) \tilde{\psi}_{k}^{a}\right\}=0
$$

From (2.18) and (2.19) we have

$$
(n-2) \tilde{\varphi}^{k} \phi_{a}\left\{S_{k}{ }^{a}+(n+2) \tilde{\varphi}_{k}^{a}\right\}=0
$$

which implies because of $n>2$ that

$$
\tilde{\psi}^{k} \psi_{a}\left\{S_{k}^{a}+(n+2) \tilde{\psi}_{k}^{a}\right\}=0 \text {. }
$$

Therefore, from the last equation, $(2.18)$ and $\phi_{a} \neq 0$ we obtain

$$
R-(n+2) \Delta=0 \text {, }
$$

which implies the following relations by $(2.17)$

$$
\begin{array}{ll}
\psi_{a}\left\{S_{k}{ }^{a}+(n+2) \bar{\psi}_{k}{ }^{a}\right\}=0, & \phi_{a}\left\{R_{j}{ }^{a}-(n+2) \phi_{j}{ }^{a}\right\}=0, \\
\bar{\psi}_{a}\left\{S_{k}{ }^{a}+(n+2) \bar{\psi}_{k}{ }^{a}\right\}=0, & \bar{\psi}_{a}\left\{R_{j}{ }^{a}-(n+2) \phi_{j}{ }^{a}\right\}=0 .
\end{array}
$$

Contracting $l$ and $h$ and transvecting with $g^{j k}$ in (2.14) and taking use of (2.20) and (2.21), we obtain

$$
\begin{aligned}
& \nabla_{a}\left\{R_{i}{ }^{a}-(n+2) \phi_{i}{ }^{a}\right\}=\bar{K}_{a}\left\{R_{i}^{a}-(n+2) \phi_{i}{ }^{a}\right\}, \\
& \nabla_{a}\left\{S_{j}{ }^{a}+(n+2) \bar{\psi}_{j}{ }^{a}\right\}=\bar{K}_{a}\left\{S_{j}{ }^{a}+(n+2) \bar{\psi}_{j}{ }^{a}\right\} .
\end{aligned}
$$

On the other hand, operating $\nabla^{i}$ to the third equation of (2.21) and taking use of (2.21) and (2.22), we have

$$
\tilde{\psi}^{a b}\left\{S_{a b}+(n+2) \bar{\psi}_{a b}\right\}=0 \text {. }
$$

From (2.3) we have

$$
\bar{\nabla}_{m} \bar{\nabla}_{l} \bar{S}_{i j}-\bar{\nabla}_{l} \bar{\nabla}_{m} \bar{S}_{i j}=0
$$

By the Ricci's identity we get

$$
\bar{S}_{a j} \bar{R}_{m l i}^{a}+\bar{S}_{i a} \bar{R}_{m l j}^{a}=0 .
$$


Using (1.12) and (1.15) we now replace $\bar{R}_{k j i}{ }^{h}$ and $\bar{S}_{j i}$ by $R_{k j i}{ }^{h}$ and $S_{j i}$ and transvect the result with $g^{l i} \bar{\psi}^{m} \psi^{j}$, then by $(2.20)$ and $(2.21)$ we have

$$
\phi_{j} \bar{\psi}^{m} R_{a l m}^{j}\left\{S^{a l}+(n+2) \tilde{\psi}^{a l}\right\}=0 \text {. }
$$

Hence, by (2.11), (2.21), (2.23) and (2.24) we get

$$
\phi_{c} \Pi_{i j k}^{c} \tilde{\psi}^{k} \Pi_{a}^{i j}{ }_{a}^{b} F_{b}^{a}=0 . \quad \text { Q. E. D. }
$$

The following corollary is trivial from our theorem.

Corollary. Let $\left(M^{n}, g, F\right)(n>2)$ be an arbitrary Kählerian space and $\left(M^{\prime n}, g^{\prime}, F^{\prime}\right)$ a locally symmetric Kählerian space. If there exists a nonaffine holomorphically projective correspondence between $\left(M^{n}, g, F\right)$ and $\left(M^{\prime n}\right.$, $\left.g^{\prime}, F^{\prime}\right)$ preserving complex structure, $\left(M^{n}, g, F\right)$ and $\left(M^{\prime n}, g^{\prime}, F^{\prime}\right)$ are of constant holomorphic sectional curvature.

\section{Department of Mathematics, Hokkaido University}

\section{References}

[1] L. P. Eisenhart : Riemannian Geometry, Princeton Univ. Press, 1949.

[2] T. ŌTSUKI and Y. TASHIRO: On curves in Kählerian space, Math. J. Okayama Univ., 4 (1954), 57-78.

[ 3 ] J. Robinson and J. D. ZUND: A theorem on geodesic mappings, Tensor (N. S), 19 (1968), 300-302.

[4] W. RoteR: Sur l'application géodésique d'une variété riemannienne sur l'espace récurrent, Bull. Acad. Polon. Sci. Sér. Sci. Math., Astr. Phys., 9 (1961), 147-149.

[5] N. S. SinYUKov: On the geodesic correspondence of a Riemannian space with a symmetric space, Dokl. Akad. Nauk. SSSR, 98 (1954), 21-23.

[6] K. YANo: Differential Geometry on Complex and Almost Complex Spaces, Pergamon Press, New York, 1965.

(Received November 28, 1973) 